\title{
PSEUDOSPECTRAL COMPONENTS AND THE DISTANCE TO UNCONTROLLABILITY*
}

\author{
J. V. BURKE ${ }^{\dagger}$, A. S. LEWIS ${ }^{\ddagger}$, AND M. L. OVERTON $^{\S}$
}

\begin{abstract}
We show that 2-norm pseudospectra of $m$-by- $n$ matrices have no more than $2 m(4 m-1)$ connected components. Such bounds are pertinent for computing the distance to uncontrollability of a control system, since this distance is the minimum value of a function whose level sets are pseudospectra. We also discuss algorithms for computing this distance, including a trisection variant of Gu's recent algorithm, and we show how these may be used to locally maximize the distance to uncontrollability for a parameterized system.
\end{abstract}

Key words. pseudospectrum, robust control, distance to uncontrollability, connected components, trisection

AMS subject classifications. Primary, 15A18, 93B05; Secondary, 65F15

DOI. $10.1137 /$ S0895479803433313

1. Introduction. For matrices $A$ and $B$ of sizes $p$-by- $p$ and $p$-by- $q$, respectively, consider the control system defined by

$$
\dot{x}=A x+B u .
$$

Here, $x \in \mathbf{R}^{p}$ is the state vector, and $u \in \mathbf{R}^{q}$ is the control vector (both depending on time). This system is controllable if, given any initial and final states $x(0)$ and $x(T)$, there exists a control function $u(\cdot)$ giving a trajectory $x(\cdot)$ with the given endpoints. In practice $A$ and $B$ are usually real.

Classical theory (see, for example, [ZDG96]) provides a simple characterization of controllability. The above system is controllable exactly when the matrix $\left[\begin{array}{lll}A-z I & B\end{array}\right]$ has full row rank for all scalars $z \in \mathbf{C}$.

Given any square matrix $A$, it is well known that the distance to the nearest singular matrix (measured in the usual operator 2-norm) is given by the smallest singular value $\sigma_{\min }(A)$ and that the conditioning of linear systems involving $A$ depends on this quantity. Another important measure is the distance from $A$ to instability, that is, the distance to the nearest matrix, possibly complex even if $A$ is real, with an eigenvalue in the closed right half-plane. This distance plays a key role in robust stability analysis of the dynamical system $\dot{x}=A x$.

The analogous question for controllability asks for the distance from the pair $(A, B)$ to the nearest pair $\left(A^{\prime}, B^{\prime}\right)$, possibly complex even if $(A, B)$ is real, corresponding to an uncontrollable system. A small distance to uncontrollability correlates with various difficulties for the control system, including numerical challenges for associated "pole placement" problems. A simple argument based on the singular value

${ }^{*}$ Received by the editors August 12, 2003; accepted for publication (in revised form) by B. T. Kågström April 8, 2004; published electronically November 17, 2004.

http://www.siam.org/journals/simax/26-2/43331.html

${ }^{\dagger}$ Department of Mathematics, University of Washington, Seattle, WA 98195 (burke@math. washington.edu, www.math.washington.edu/ burke). The research of this author was supported in part by National Science Foundation grant DMS-9971852.

${ }^{\ddagger}$ Department of Mathematics, Simon Fraser University, Burnaby, BC V5A 1S6, Canada (aslewis@ sfu.ca, www.cecm.sfu.ca/ aslewis). The research of this author was supported in part by NSERC.

$\S$ Courant Institute of Mathematical Sciences, New York University, New York, NY 10012 (overton @cs.nyu.edu, www.cs.nyu.edu/ overton). The research of this author was supported in part by National Science Foundation grant CCR-0098145. 


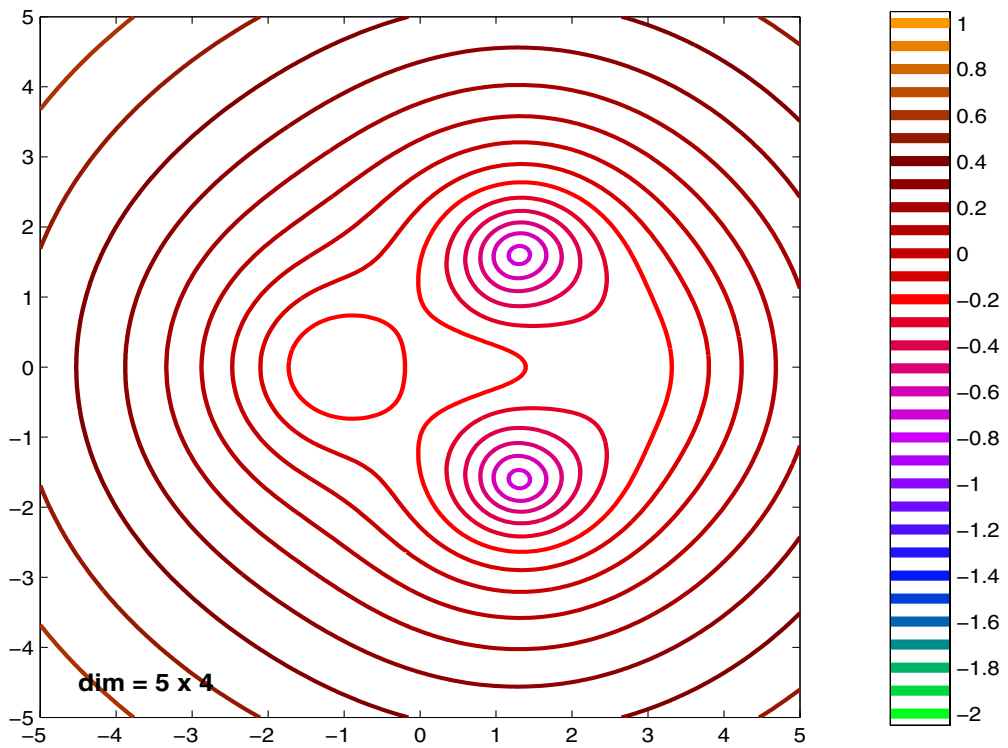

FIG. 1. Pseudospectra for the controllable pair (1.2) with $x_{1}=x_{2}=1$.

decomposition [Eis84] shows that the distance to uncontrollability is given by

$$
\min _{z \in \mathbf{C}} \sigma_{\min }[A-z I \quad B]
$$

a global optimization problem in two real variables. Here $\sigma_{\min }$ of a $p$-by- $p+q$ matrix $C$ means the square root of the smallest eigenvalue of $C C^{*}$, a positive quantity when $C$ has rank $p$.

The function to be minimized in the expression (1.1) has lower level sets of the form

$$
\left\{z \in \mathbf{C}: \sigma_{\min }[A-z I \quad B] \leq \epsilon\right\}
$$

for real $\epsilon>0$. These sets, commonly called pseudospectra, have been well studied for square matrices, when the matrix $B$ is empty; see the Pseudospectra Gateway [ET]. Pseudospectra are less well understood in the rectangular case, but references include [TT96, WT01, HT02, WT02, BEGM03]. Substantial insight is gained from examples, so consider the parameterized matrix pair

$$
(A, B)\left(x_{1}, x_{2}\right)=\left(\left[\begin{array}{cccc}
1 & 1 & 2 & 3 \\
-1 & 1 & 4 & 5 \\
0 & x_{1} & 1 & 2 \\
x_{2} & 0 & -2 & 1
\end{array}\right],\left[\begin{array}{l}
1 \\
1 \\
0 \\
0
\end{array}\right]\right)
$$

where $x_{1}$ and $x_{2}$ are real parameters. Figures 1 and 2 show pseudospectra ${ }^{1}$ for, respectively, the controllable pair (1.2) when $x_{1}=x_{2}=1$ and the uncontrollable pair (1.2) when $x_{1}=x_{2}=0$ (the latter case being an example from [Gu00]).

The horizontal and vertical axes in the figures show the real and imaginary parts of $z$. The legends on the right sides of the figures show the contour heights (values

\footnotetext{
${ }^{1}$ All the figures in this paper were produced using T. Wright's software EigTool [Wri02].
} 


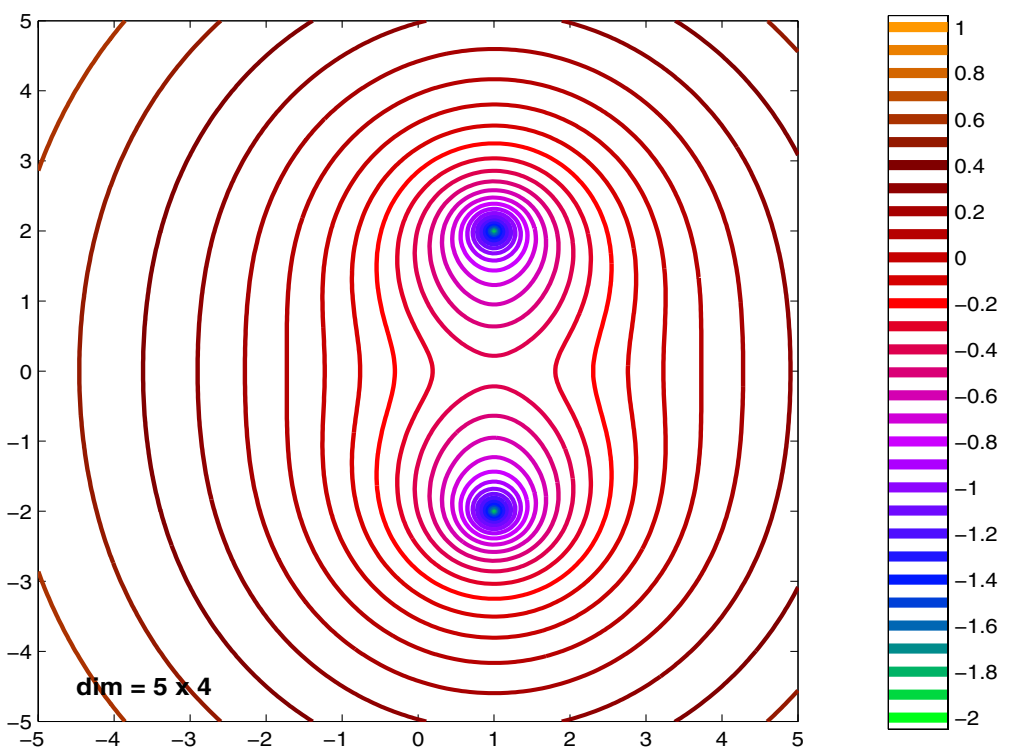

FIG. 2. Pseudospectra for the uncontrollable pair (1.2) with $x_{1}=x_{2}=0$.

of $\epsilon$ ) on a $\log 10$ scale, with both plots using the same scale. In Figure 1, the "pseudospectral landscape" has three local minimizers and one can estimate that the global minimum value (by definition, the distance to uncontrollability) is about $10^{-0.7}$ (in fact, it is 0.1872). In Figure 2, there are only two local minimizers (forming a complex conjugate pair), and one can see that the contours drop to much lower values (in fact, it is easy to check that the minimum value of (1.1) is zero at the points $z=1 \pm 2 i$ ). In Figure 2 it is clear that some pseudospectra contain two connected components. In Figure 1, it is not clear, without a more detailed analysis, whether there are values of $\epsilon$ for which the pseudospectra have three connected components (in fact, there do exist such $\epsilon$ ).

Our aim in this work is to find an upper bound on the number of connected components in the pseudospectra of rectangular matrices. We use a slightly more general setting than described above, defining

$$
\Lambda=\left\{z \in \mathbf{C}: \sigma_{\min }(P+z Q) \leq \epsilon\right\}
$$

for given matrices $P$ and $Q$ in the space $\mathbf{M}^{m, n}$ of $m$-by- $n$ complex matrices (with $m \leq n)$. In the case above we have $P=[A B]$ and $Q=\left[\begin{array}{ll}-I & 0\end{array}\right]$. Our goal is to find an upper bound on the number of components of the set $\Lambda$. Specifically, we show this number is no more than $2 m(4 m-1)$. To our knowledge, this general bound is the best known, although it is certainly not tight. In particular, it is well known that pseudospectra of $m$-by- $m$ matrices have no more than $m$ components, since each component contains an eigenvalue [Tre97]. Furthermore, in the case of a single row $(m=1)$, it is easy to see that each nonempty pseudospectrum is simply a circular disk. We are not aware of an example of a pseudospectrum with more than $m$ components.

We hope our analysis of pseudospectral components will shed light on the complexity of the problem of computing the distance to uncontrollability, for which we discuss algorithms in the second half of the paper. We begin by discussing a recent algorithm due to $\mathrm{Gu}[\mathrm{Gu} 00]$ for estimating the distance to uncontrollability within 
a factor of two, and we show how a trisection variant can be used to obtain any prescribed accuracy. We then discuss an algorithm that combines repeated local optimization with Gu's algorithm and speculate that techniques similar to those used in analyzing the number of pseudospectral components might be used to bound the number of local optimization steps in this process.

Finally, with an effective algorithm in hand to evaluate the distance to uncontrollability (and, where defined, its gradient), we consider local maximization of the distance to uncontrollability for a smoothly varying parameterized pair $(A, B)$ over a vector of free parameters. For the family (1.2), we find a locally maximizing pair with pseudospectra having four components.

2. Generic properties of singular values. To prove an upper bound on the number of components of the set $\Lambda$ defined by (1.3), we first dispose of some trivial cases. Clearly we can suppose $Q$ is nonzero, and hence $\Lambda$ is compact. Furthermore, only the case $\epsilon \geq 0$ is interesting, as otherwise $\Lambda$ is empty.

When $\epsilon=0$, the set $\Lambda$ either is the whole complex plane or consists of at most $m$ points, as the following argument shows. Notice $\Lambda$ is just the set of complex $z$ for which $P+z Q$ has rank less than $m$. Assuming $\Lambda$ is not the whole plane, we lose no generality in supposing that it does not contain zero or, in other words, that the matrix $P$ has rank $m$. Partition the matrices $P$ and $Q$ as $\left[\begin{array}{ll}P_{1} & P_{2}\end{array}\right]$ and $\left[\begin{array}{ll}Q_{1} & Q_{2}\end{array}\right]$, respectively, where $P_{1}$ and $Q_{1}$ are $m$-by- $m$, and, again without loss of generality, $P_{1}$ is invertible. Since the function $\operatorname{det}\left(P_{1}+z Q_{1}\right)$ is a polynomial of degree at most $m$, and is nonzero at zero, it has at most $m$ zeros. But this set of zeros contains $\Lambda$, so the claim follows.

Henceforth we therefore assume $\epsilon>0$. In the case $m=1$, an easy calculation shows that $\Lambda$ is either empty or a circular disk.

Our goal in this section is to show that for a fixed $\delta>0$ and a "generic" matrix $P$, the singular value $\sigma_{\min }(P+z Q)$ is always either simple or less than $\delta$. The proof is based on the following classical result in the space of $m$-by- $m$ Hermitian matrices $\mathbf{H}^{m}$ (a real vector space of dimension $m^{2}$ ), concerning matrices $X$ with a multiple smallest eigenvalue $\lambda_{\min }(X)$.

Theorem 2.1 (von Neumann and Wigner [vNW29]). For any integer $m>1$, the algebraic set

$$
\hat{\mathbf{H}}^{m}=\left\{X \in \mathbf{H}^{m}: \lambda_{\min }(X) \text { multiple }\right\}
$$

has real codimension 3 .

For example, the space $\mathbf{H}^{2}$ has dimension 4 , and the set $\hat{\mathbf{H}}^{2}$ consists simply of real multiples of the identity matrix.

We also need an elementary supporting result.

Proposition 2.2 (surjectivity). A matrix $Y \in \mathbf{M}^{m, n}$ has full row rank if and only if the function $X \mapsto X Y^{*}+Y X^{*}$ maps $\mathbf{M}^{m, n}$ onto $\mathbf{H}^{m}$.

Proof. Denote the given function by $\Phi: \mathbf{M}^{m, n} \rightarrow \mathbf{H}^{m}$. If $Y$ has full row rank, then, with no loss of generality, $Y=\left[\begin{array}{ll}Y_{0} & Y_{1}\end{array}\right]$, where the matrix $Y_{0}$ is invertible. Now, given any matrix $E \in \mathbf{H}^{m}$, we have $\Phi\left(\frac{1}{2}\left[E Y_{0}^{-*} 0\right]\right)=E$, so $\Phi$ is indeed onto.

Conversely, suppose the map $\Phi$ is onto, and some $x \in \mathbf{C}^{m}$ satisfies $Y^{*} x=0$. Choose a matrix $X \in \mathbf{H}^{m}$ satisfying $\Phi(X)=x x^{*}$. Then

$$
\|x\|^{4}=x^{*}\left(X Y^{*}+Y X^{*}\right) x=0,
$$

so $x=0$, as required. 
We are now ready for the main result of this section.

Theorem 2.3 (generic singular values). For any $n \geq m>1$ and any real $\delta>0$, the real semialgebraic set

$$
\left\{Y \in \mathbf{M}^{m, n}: \sigma_{\min }(Y) \text { is both multiple and at least } \delta\right\}
$$

has real codimension 3 .

Proof. Define a map $\Psi: \mathbf{M}^{m, n} \rightarrow \mathbf{H}^{m}$ by $\Psi(Y)=Y Y^{*}$. Notice that the given set, which we denote $S$, is defined locally by the inverse image $\Psi^{-1}\left(\hat{\mathbf{H}}^{m}\right)$. Furthermore, any $Y \in S$ has full row rank, and so Proposition 2.2 shows that the derivative $\nabla \Psi(Y)$ is onto. Since $\hat{\mathbf{H}}^{m}$ has codimension 3 by the result of von Neumann and Wigner (Theorem 1.1), so does $S$.

Corollary 2.4. For any $n \geq m>1$, real $\delta>0$, and matrix $Q \in \mathbf{M}^{m, n}$, the real semialgebraic set

$$
\left\{P \in \mathbf{M}^{m, n}: \exists z \in \mathbf{C} \text { so } \sigma_{\min }(P+z Q) \text { is both multiple and at least } \delta\right\}
$$

has real codimension at least 1 .

It follows from this last corollary that for a generic matrix $P$, the singular value $\sigma_{\min }(P+z Q)$ is always either simple or less than $\delta$.

3. The generic case. Our bound on the number of components of pseudospectra relies on the following classical result [Mil64].

Theorem 3.1 (Milnor). For any polynomial $p: \mathbf{R}^{2} \rightarrow \mathbf{R}$ of degree $d$, the zero set $p^{-1}(0)$ has no more than $d(2 d-1)$ components.

(In fact Milnor bounds the sum of the Betti numbers of $p^{-1}(0)$ : the result above follows from the fact that the number of components is the zeroth Betti number.)

To apply Milnor's result, we need to relate the number of components of pseudospectra to their boundaries. We accomplish this with the following elementary result.

Proposition 3.2 (components and boundaries). Consider any continuous function $f: \mathbf{C} \rightarrow \mathbf{R}$. If the zero set $f^{-1}(0)$ is nonempty, then it has at least as many components as the level set $f^{-1}(-\infty, 0]$.

Proof. Denote the zero set by $E$ and the level set by $L$. It suffices to show that every component of $L$ contains a component of $E$. If this is not the case, $L$ has a component $L_{1}$ contained in the set $L^{\prime}=f^{-1}((-\infty, 0))$. By continuity, $L$ is closed and $L^{\prime}$ is open. Hence $L_{1}$, which is a component of both sets, must be both closed and open, and hence equal to the whole complex plane. Thus $E$ must be empty, contrary to assumption.

Using this technique in conjunction with Milnor's theorem, we can now prove our basic result.

TheOREM 3.3 (generic case). Given any real $\epsilon$ and matrices $P, Q \in \mathbf{M}^{m, n}$ (where $m \leq n)$, suppose there exists no complex $z$ for which the singular value $\sigma_{\min }(P+z Q)$ is both multiple and equals $\epsilon$. Then the set

$$
\Lambda=\left\{z \in \mathbf{C}: \sigma_{\min }(P+z Q) \leq \epsilon\right\}
$$

has no more than $2 m(4 m-1)$ components.

Proof. The case $m=1$ is elementary, so we suppose $m>1$. For any matrix $A \in \mathbf{M}^{m, n}$, we write the singular values of $A$ by multiplicity and in decreasing order: $\sigma_{1}(A) \geq \sigma_{2}(A) \geq \cdots \geq \sigma_{m}(A)$. In this notation, $\sigma_{\min }(A)=\sigma_{m}(A)$. 
Consider the two disjoint open sets

$$
\begin{aligned}
& \Gamma_{<}=\left\{z \in \mathbf{C}:\left(\sigma_{m}+\sigma_{m-1}\right)(P+z Q)<2 \epsilon\right\}, \\
& \Gamma_{>}=\left\{z \in \mathbf{C}:\left(\sigma_{m}+\sigma_{m-1}\right)(P+z Q)>2 \epsilon\right\} .
\end{aligned}
$$

By assumption, the set

$$
\Lambda^{\prime}=\left\{z \in \mathbf{C}: \sigma_{\min }(P+z Q)=\epsilon\right\}
$$

is contained in $\Gamma_{>}$, whereas the set

$$
\Lambda^{\prime \prime}=\bigcup_{j=1}^{m-1}\left\{z \in \mathbf{C}: \sigma_{j}(P+z Q)=\epsilon\right\}
$$

is contained in $\Gamma_{<}$. Hence $\Lambda^{\prime}$ has no more components than the set

$$
\begin{aligned}
\Lambda^{\prime} \cup \Lambda^{\prime \prime} & =\bigcup_{j=1}^{m}\left\{z \in \mathbf{C}: \sigma_{j}(P+z Q)=\epsilon\right\} \\
& =\left\{z \in \mathbf{C}: \operatorname{det}\left((P+z Q)(P+z Q)^{*}-\epsilon^{2} I\right)=0\right\} .
\end{aligned}
$$

We can suppose that the matrix $Q$ is nonzero and that the set $\Lambda$ is nonempty. Applying Proposition 3.2 (components and boundaries) to the function $f(z)=\sigma_{\min }(P+$ $z Q)-\epsilon$ shows that $\Lambda$ has no more components than $\Lambda^{\prime}$, and hence no more than $\Lambda^{\prime} \cup \Lambda^{\prime \prime}$.

The function $\phi: \mathbf{C}^{2} \rightarrow \mathbf{C}$ defined by

$$
\phi(x, y)=\operatorname{det}\left((P+(x+i y) Q)(P+(x+i y) Q)^{*}-\epsilon^{2} I\right)
$$

is clearly a polynomial of degree $2 \mathrm{~m}$. Since Hermitian matrices have real determinants, $\phi(x, y)$ is real for all real $x$ and $y$. Thus the restriction $\left.\phi\right|_{\mathbf{R}^{2}}$ is a polynomial of degree $2 m$ (whose coefficients we could identify by partial differentiation). The zero set of this polynomial is

$$
\left\{(x, y) \in \mathbf{R}^{2}: x+i y \in \Lambda^{\prime} \cup \Lambda^{\prime \prime}\right\},
$$

and our result now follows by applying Milnor's theorem (3.1).

4. The general case. We extend our basic result, Theorem 3.3 (generic case), to the general case by a limiting argument. Recall that a sequence of subsets $S_{r}$ of some Euclidean space converges to another set $S$ if the following properties hold [RW98]:

(i) For any point $x \in S$, there exists a sequence of points $x_{r} \in S_{r}$ whose limit is $x$.

(ii) For any subsequence $R$ of $\mathbf{N}$, the limit of any convergent sequence of points $x_{r} \in S_{r}(r \in R)$ lies in $S$.

We first prove that, with this notion of convergence, the number of components of a compact set has a lower semicontinuity property.

Proposition 4.1 (lower semicontinuity). Consider a sequence of closed subsets of $S_{r}$ of a Euclidean space converging to a compact set $S$. If $S$ has a finite number of components, say $k$, then $S_{r}$ has at least $k$ components for all large $r$. 
Proof. We can suppose the set $S$ is nonempty. Denote its components by $S^{j}$ $(j=1,2, \ldots, k)$, and the closed and open unit balls by $B$ and $B^{\circ}$, respectively. Components of compact sets are compact, so for some real $\delta>0$, the sets $S^{j}+\delta B$ $(j=1,2, \ldots, k)$ are disjoint. Choose real $M$ so that $S+\delta B \subset M B$.

We first claim

$$
S_{r} \subset\left(S+\delta B^{\circ}\right) \cup M B^{c} \text { for all large } r .
$$

Otherwise there would be a subsequence $R$ of $\mathbf{N}$ and points

$$
x_{r} \in S_{r} \cap\left(S+\delta B^{\circ}\right)^{c} \cap M B \quad(r \in R) .
$$

This bounded sequence has a cluster point in the closed set $\left(S+\delta B^{\circ}\right)^{c}$, contradicting the fact that the sets $S_{r}$ converge to $S$.

Thus for all large $r$, the set $S_{r}$ is contained in the disjoint union of open sets

$$
M B^{c} \cup \bigcup_{j=1}^{k}\left(S^{j}+\delta B^{\circ}\right) .
$$

If the result fails, then the number of components of $S_{r}$ is strictly less than $k$ for all $r$ in some subsequence $R$ of $\mathbf{N}$. Hence for some index $j$ and a further subsequence $R^{\prime}$ of $R$, we must have

$$
S_{r} \cap\left(S^{j}+\delta B^{\circ}\right)=\emptyset \quad \text { for all } r \in R^{\prime} .
$$

But this contradicts the definition of convergence, since for any point $x \in S^{j}$ there exists a sequence of points $x_{r} \in S_{r}$ converging to $x$.

Using this result, we can prove our main result.

TheOREM 4.2 (components of pseudospectra). For any matrices $P, Q \in \mathbf{M}^{m, n}$ (where $m \leq n$ ) and any real $\epsilon$, the set $\left\{z \in \mathbf{C}: \sigma_{\min }(P+z Q) \leq \epsilon\right\}$ has no more than $2 m(4 m-1)$ components.

Proof. We can suppose that the given set, which we denote by $\Lambda$, is nonempty, that $\epsilon>0$, and that $Q$ is nonzero.

By Corollary 2.4, there exists a sequence of matrices $P_{r} \in \mathbf{M}^{m, n}$ satisfying the following two conditions:

(i) $\left\|P_{r}-P\right\| \leq 1 / r$.

(ii) For no $z \in \mathbf{C}$ is $\sigma_{\min }\left(P_{r}+z Q\right)$ both multiple and equal to $\epsilon+1 / r$.

It follows by Theorem 3.3 (generic case) that the set

$$
\Lambda_{r}=\left\{z \in \mathbf{C}: \sigma_{\min }\left(P_{r}+z Q\right) \leq \epsilon+\frac{1}{r}\right\}
$$

has no more than $2 m(4 m-1)$ components.

Using a well-known property of singular values, any point $z \in \Lambda$ satisfies

$$
\sigma_{\min }\left(P_{r}+z Q\right) \leq \sigma_{1}\left(P_{r}-P\right)+\sigma_{\min }(P+z Q) \leq \frac{1}{r}+\epsilon,
$$

so $\Lambda \subset \Lambda_{r}$ for all $r$. On the other hand, the continuity of $\sigma_{\text {min }}$ shows that any cluster point of a sequence of points $z_{r} \in \Lambda_{r}$ must lie in $\Lambda$. Thus the compact sets $\Lambda_{r}$ converge to the compact set $\Lambda$.

Finally, notice that $\Lambda$, being semialgebraic, has finitely many components. Hence we can apply Proposition 4.1 (lower semicontinuity) to deduce that, in fact, it has no more than $2 m(4 m-1)$ components, as required. 
5. Computing the distance to uncontrollability. Let $\tau(A, B)$ denote the distance to uncontrollability for a pair $(A, B)$, defined by (1.1), where $A$ is $p$-by- $p$ and $B$ is $p$-by-q. Thus the problem of computing $\tau(A, B)$ is that of minimizing $\sigma_{\min }\left[\begin{array}{lll}A-z I & B\end{array}\right]$ over the whole complex plane, a global minimization problem in two real variables.

It is interesting to compare the difficulty of this problem with that of two others: computing the distance to singularity and the distance to instability for the $p$-by- $p$ matrix $A$ alone. Let us assume that the computation of the minimum singular value function $\sigma_{\min }$ is an atomic operation. Computing the distance to singularity (distance to the nearest singular matrix) then requires one evaluation of $\sigma_{\min }$, while the distance to instability (distance to the nearest unstable matrix) may be computed by minimizing $\sigma_{\min }(A-z I)$ over the imaginary axis (equivalently, a global optimization problem in one real variable). Computation of the distance to instability, say $\beta(A)$, is a standard operation in control. The key observation is that checking whether $\beta(A)$ is less than a fixed number $\delta$ simply requires checking whether an associated Hamiltonian matrix has any imaginary eigenvalues. This leads immediately to a bisection algorithm [Bye88, BS90] that evaluates $\beta(A)$ to any prescribed accuracy in exact arithmetic, taking the computation of eigenvalues of $2 p$-by- $2 p$ Hamiltonian matrices as another atomic operation. Higher-order convergent algorithms are also well known [BB90]. In practice, it is important to compute the eigenvalues of the Hamiltonian matrices by a special algorithm that preserves Hamiltonian structure (such as in [Van84]) in order to avoid numerical blunders that incorrectly identify an eigenvalue as nonimaginary because of unnecessary rounding errors in its real part. The Hamiltonian imaginary eigenvalue test in these algorithms may be replaced by a linear matrix inequality (LMI) test (see, e.g., [BTN01]). This is computationally more expensive in practice, but offers the advantage of a complexity analysis that does not require assumption of eigenvalue and singular value computation as atomic operations.

By contrast, computing the distance to uncontrollability $\tau(A, B)$ seems to be a harder problem, and there are no standard methods in use, though there have been some recent theoretical advances. In 2000, Gu published an algorithm [Gu00] that estimates $\tau(A, B)$ within a factor of two. Gu's algorithm is based on a most ingenious test ("Gu's test," for brevity) that compares imaginary eigenvalues of matrix pencils involving Kronecker products that depend on $A$ and $B$. Taking the computation of singular values and eigenvalues as atomic operations that can be performed in time cubic in the matrix dimension, and assuming that $q=O(p)$ (in practice, typically $q<p)$, Gu's test requires $O\left(p^{6}\right)$ operations. No other polynomial-time algorithm for estimating $\tau(A, B)$ within a constant factor seems to be known; in particular, it does not seem to be known whether Gu's test could be replaced by an LMI-based test.

Gu's test may be summarized as follows. Given two numbers $\delta_{1}$ and $\delta_{2}$ (known as $\delta$ and $\delta-\eta / 2$, respectively, in [Gu00]), with $\delta_{1}>\delta_{2}>0$, Gu's test returns either the information that

$$
\tau(A, B) \leq \delta_{1}
$$

or the information that

$$
\tau(A, B)>\delta_{2}
$$

At least one of these statements must be true; even if both are true, only one of the two statements is verified. As already noted, Gu's test involves comparing imaginary 
eigenvalues of matrix pencils. We note for the record that both the terms $Q_{12} \otimes A$ and $I \otimes\left(A^{*} Q_{12}\right)$ in the definition of $\mathcal{A}$ in [Gu00, p. 996] have incorrect signs.

Gu's estimation algorithm is then as follows.

Algorithm 5.1 (Gu's estimation algorithm).

1. Set $\delta_{1}=\sigma_{\min }\left(\left[\begin{array}{ll}A & B\end{array}\right]\right)$, done $=$ false.

2. While not done

(a) $\operatorname{Set} \delta_{2}=\delta_{1} / 2$.

(b) Perform Gu's test. If (5.1) is verified, set $\delta_{1}=\delta_{2}$; if (5.2) is verified, set done $=$ true.

In exact arithmetic, this algorithm evaluates a nonzero $\tau(A, B)$ within a factor of two, but does not terminate if $\tau(A, B)=0$.

It is tempting to try to evaluate $\tau(A, B)$ to higher precision by a bisection method. In order to make this work, one needs to set $\delta_{1}$ and $\delta_{2}$ sufficiently close to each other ( $\eta$ sufficiently small in the notation of [Gu00]) that (5.1) and (5.2) are almost mutually exclusive. Unfortunately, this leads to numerical difficulties; the necessary comparison of imaginary eigenvalues of the relevant pencils cannot be carried out with any confidence in the presence of rounding errors. However, a trisection variant works well, as follows.

Algorithm 5.2 (trisection variant of Gu's algorithm).

1. Set $L=0, U=\sigma_{\min }\left(\left[\begin{array}{ll}A & B\end{array}\right]\right)$, done $=$ false, tol to a positive tolerance.

2. While not done

(a) Set $\delta_{1}=L+2(U-L) / 3$ and $\delta_{2}=L+(U-L) / 3$.

(b) Perform Gu's test. If (5.1) is verified, set $U=\delta_{1}$; if (5.2) is verified, set $L=\delta_{2}$.

(c) If $U-L<$ tol, set done $=$ true.

This trisection algorithm maintains upper and lower bounds $U$ and $L$ on $\tau(A, B)$, reducing the length of the interval $[L, U]$ by a factor of $2 / 3$ at each step of the iteration, and thereby computing $\tau(A, B)$ to any prescribed absolute accuracy in exact arithmetic in $O\left(p^{6}\right)$ operations. Furthermore, it is effective in practice even in the presence of rounding errors, running into numerical trouble only when $\tau(A, B)$ is determined at least to a few accurate digits.

An algorithm that runs much faster in practice, but without any complexity guarantee, is based on local optimization. This algorithm repeatedly performs local optimization of (1.1) using, for example, the well-known BFGS method. For controllable pairs, one expects the objective in (1.1) to be differentiable at minimizers, since the least singular value is being minimized, not maximized. As long as the least singular value at a local minimizer is simple and nonzero, the objective in (1.1) is continuously differentiable there. The BFGS algorithm requires the gradient of $\sigma_{\min }\left[\begin{array}{ll}A-z I & B\end{array}\right]$ with respect to the real and imaginary parts of $z$, which is given by

$$
\left[\begin{array}{l}
\operatorname{Re}\left(([I 0] u)^{*} v\right) \\
\operatorname{Im}\left(([I 0] u)^{*} v\right)
\end{array}\right]
$$

where $u$ and $v$ are, respectively, the left and right singular vectors corresponding to $\sigma_{\min }\left[\begin{array}{ll}A-z I & B\end{array}\right]$. (One could use Newton's method instead of BFGS, as the corresponding 2-by-2 Hessian matrix is not hard to derive, but BFGS is so fast that Newton's method offers no significant advantage.) Once a local minimizer is obtained, Gu's test is used either to (i) verify global optimality within a tolerance or (ii) restart BFGS. A key point is that when Gu's test verifies (5.1), it also provides $\hat{z}$ for which $\sigma_{\min }[A-\hat{z} I \quad B]=\delta_{1}$. 
Algorithm 5.3 (BFGS/Gu hybrid).

1. Set $U=\sigma_{\min }([A B]), z=0$, done $=$ false, tol to a positive tolerance.

2. While not done

(a) Run BFGS starting at $z$, producing an approximate local minimizer $\tilde{z}$. Set $\tilde{f}=\sigma_{\min }[A-\tilde{z} I B]$.

(b) Set $\delta_{1}=\tilde{f}(1-0.5$ tol $)$ and $\delta_{2}=\tilde{f}(1-$ tol $)$.

(c) Perform Gu's test. If (5.1) is verified, set $U=\delta_{1}$ and $z=\hat{z}$, where $\sigma_{\min }\left[\begin{array}{lll}A-\hat{z} I & B\end{array}\right]=\delta_{1}$; if (5.2) is verified, set $L=\delta_{2}$ and done $=$ true.

Although the objective function in (1.1) may have infinitely many local minimizers [GdH99], it has only finitely many locally minimal values (being semialgebraic). Assuming that an idealized BFGS algorithm always generates an exact local minimizer, in exact arithmetic the BFGS/Gu hybrid is guaranteed to terminate with an estimate of a nonzero $\tau(A, B)$ within any prescribed relative accuracy. A natural question is: how many local minimizers might be visited before a global optimizer is obtained? Unfortunately, our bound on the number of connected components does not immediately yield a bound on the number of locally minimal values. Nonetheless, we think that it might be possible to obtain a bound on the latter quantity by extending the techniques used to bound the former.

Our Matlab implementations of the algorithms described in this section are freely available. ${ }^{2}$

6. Maximizing the distance to uncontrollability for a parameterized matrix pair. Finally, with two effective algorithms to evaluate $\tau(A, B)$ available, namely, the trisection variant of Gu's algorithm and the BFGS/Gu hybrid, we consider maximization of the distance to uncontrollability for a smoothly varying parameterized pair $(A, B)(x)$ over a vector of free parameters $x \in \mathbf{R}^{k}$. There are two reasons why this might be of interest. The first is that it may well be useful in applications to be able to find a matrix pair that is optimally far away from uncontrollability with respect to given free parameters. The second reason is that maximizing the distance to uncontrollability tends to produce pseudospectra with several isolated local minimizers whose minimal values are equal, and therefore is likely to produce pseudospectra with more components than would be found by randomly generating matrix pairs.

It is not difficult to see that the function $\tau(\cdot)$ is not differentiable on the space of (real or complex) matrix pairs; furthermore, it is easy to construct parameterized examples where the composite parameter-dependent function $\tau((A, B)(\cdot))$ is not differentiable at its maximizer. Such functions are not amenable to optimization by standard methods, such as BFGS, so we use a more specialized "gradient sampling" algorithm [BLO03]. This method exploits the fact that $\tau(\cdot)$ is differentiable almost everywhere, with gradient given by $u v^{*}$, where $u$ and $v$ are, respectively, the relevant left and right singular vectors for the matrix minimizing $\sigma_{\min }[A-z I B]$ over $z$, as long as the minimum singular value is simple and nonzero. We omit further details here and conclude by considering the example in (1.2). Running the gradient sampling algorithm to locally maximize the distance to uncontrollability over $x_{1}$ and $x_{2}$, we found an approximate local maximizer $\hat{x}$ given by $\hat{x}_{1}=1.9787, \hat{x}_{2}=-1.8667$, with $\hat{\tau}=\tau((A, B)(\hat{x}))=0.4897$. The corresponding pseudospectra are shown in Figure 3 . Notice that the lowest points in this "pseudospectral landscape" are higher than those in Figure 1 and, furthermore, that four local minimizers have the same minimal value (namely, $\hat{\tau}$ ). Only two of the local minimizers occur in a complex conjugate pair; the

${ }^{2}$ http://www.cs.nyu.edu/faculty/overton/software 


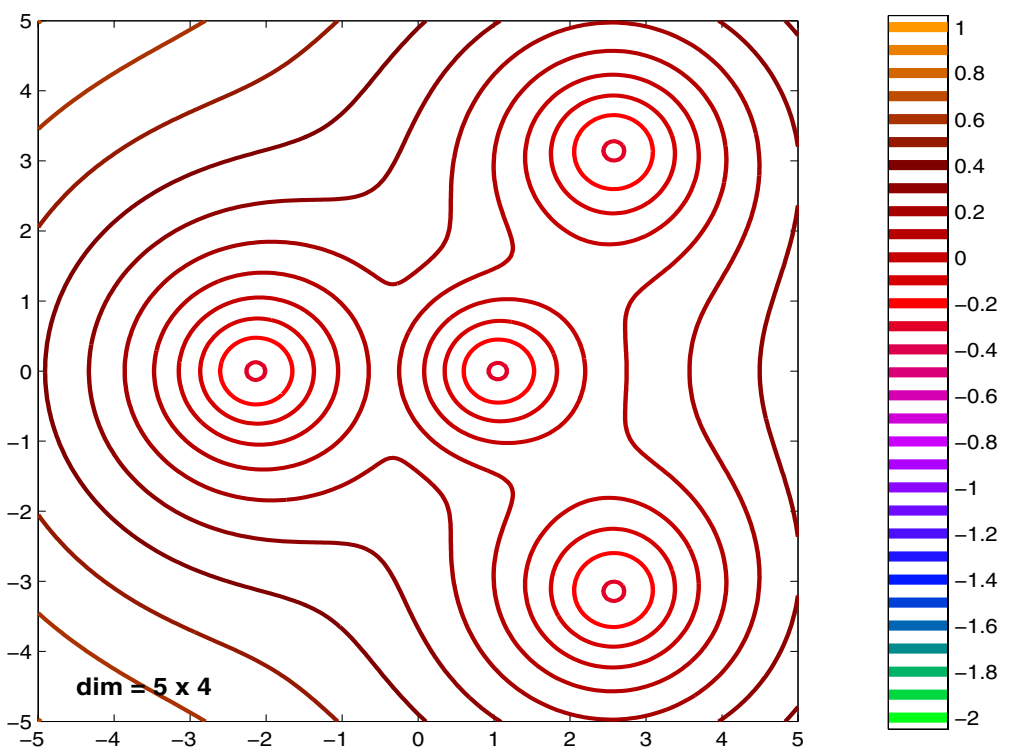

FIG. 3. Pseudospectra for a local maximizer of $\tau$ over (1.2).

other "ties" occur as a result of optimization over the parameters, with $\tau((A, B)(\cdot))$ not differentiable at its maximizer $\hat{x}$ as a result. Since there are four isolated local minimizers with minimal value $\hat{\tau}$, it follows that the pseudospectra have four components for $\epsilon$ in a range above $\hat{\tau}$. In this example, the row dimension $p$ in fact equals four. Whether it is possible to produce pseudospectra with more than $p$ components remains an open question.

Note added in proof. In fact, the bound $d(2 d-1)$ in Milnor's result (Theorem 3.1) can be replaced by the sharp bound $\left(d^{2}-d+2\right) / 2$ [BR90, Exercise 4.4.4], resulting in the improvement of our bound in Theorem 4.2 from $2 m(4 m-1)$ to $2 m^{2}-m+1$. Whether a subquadratic bound holds is still an open question.

Acknowledgments. Many thanks to Nick Trefethen for posing the question of how to bound the number of pseudospectral components and for various helpful comments on this paper. Many thanks also to Ricky Pollack for pointing out Milnor's result.

\section{REFERENCES}

[BB90] S. Boyd And V. BALAKRishnan, A regularity result for the singular values of a transfer matrix and a quadratically convergent algorithm for computing its $L_{\infty}$-norm, Systems Control Lett., 15 (1990), pp. 1-7.

[BEGM03] B. Boutry, M. Elad, G.H. Golub, and P. Milanfar, The generalized eigenvalue problem for nonsquare pencils using a minimal perturbation approach, SIAM J. Matrix Anal. Appl., to appear.

[BLO03] J.V. Burke, A.S. Lewis, AND M.L. Overton, A robust gradient sampling algorithm for nonsmooth, nonconvex optimization, SIAM J. Optim., to appear.

[BR90] R. Benedetti And J.-J. Risler, Real Algebraic and Semi-algebraic Sets, Hermann, Paris, 1990.

[BS90] N.A. Bruinsma And M. Steinbuch, A fast algorithm to compute the $\mathbf{H}_{\infty}$-norm of a transfer function matrix, Systems Control Lett., 14 (1990), pp. 287-293. 
[BTN01] A. Ben-Tal and A. Nemirovski, Lectures on Modern Convex Optimization: Analysis, Algorithms, and Engineering Applications, MPS/SIAM Ser. Optim. 2, SIAM, Philadelphia, 2001.

[Bye88] R. ByERs, A bisection method for measuring the distance of a stable matrix to the unstable matrices, SIAM J. Sci. Statist. Comput., 9 (1988), pp. 875-881.

[Eis84] R. EIsIng, Between controllable and uncontrollable, Systems Control Lett., 4 (1984), pp. 263-264.

[ET] M. Embree And L.N. Trefethen, Pseudospectra Gateway, http://web.comlab.ox.ac. uk/projects/pseudospectra/.

[GdH99] J.-M. GRACIA AND I. DE HoYOs, Nearest pairs with more nonconstant invariant factors and pseudospectra, Linear Algebra Appl., 298 (1999), pp. 143-158.

[Gu00] M. Gu, New methods for estimating the distance to uncontrollability, SIAM J. Matrix Anal. Appl., 21 (2000), pp. 989-1003.

[HT02] N.J. Higham AND F. TisseuR, More on pseudospectra for polynomial eigenvalue problems and applications in control theory, Linear Algebra Appl., 351/352 (2002), pp. 435-453.

[Mil64] J.W. Milnor, On the Betti numbers of real varieties, Proc. Amer. Math. Soc., 15 (1964), pp. 275-280.

[RW98] R.T. Rockafellar and R.J.-B. Wets, Variational Analysis, Springer, Berlin, 1998.

[Tre97] L.N. Trefethen, Pseudospectra of linear operators, SIAM Rev., 39 (1997), pp. 383-406.

[TT96] K.-C. Toh And L.N. Trefethen, Calculation of pseudospectra by the Arnoldi iteration, SIAM J. Sci. Comput., 17 (1996), pp. 1-15.

[Van84] C. VAN LOAN, A symplectic method for approximating all the eigenvalues of a Hamiltonian matrix, Linear Algebra Appl., 61 (1984), pp. 233-251.

[vNW29] J. von Neumann And E. Wigner, Über das Verhalten von Eigenwerten bei adiabatischen Prozessen, Physik. Zeitschr., 30 (1929), pp. 467-470.

[Wri02] T.G. Wright, EigTool: A Graphical Tool for Nonsymmetric Eigenproblems, 2002; available online from http://web.comlab.ox.ac.uk/pseudospectra/eigtool/.

[WT01] T.G. WRIGHT AND L.N. TREFEThen, Large-scale computation of pseudospectra using ARPACK and eigs, SIAM J. Sci. Comput., 23 (2001), pp. 591-605.

[WT02] T.G. Wright and L.N. Trefethen, Pseudospectra of rectangular matrices, IMA J. Numer. Anal., 22 (2002), pp. 501-519.

[ZDG96] K. Zhou, J.C. Doyle, and K. Glover, Robust and Optimal Control, Prentice-Hall, Upper Saddle River, NJ, 1996. 